\title{
Correction to: Positional behavior, habitat use, and forelimb morphology of Père David's Rock Squirrels Sciurotamias davidianus (Milne-Edwards, 1867) (Sciuridae, Rodentia) in the Qinling Mountains, Shaanxi, China
}

\author{
Dionisios Youlatos ${ }^{1}\left[\mathrm{C} \cdot \mathrm{Gang} \mathrm{He} \mathrm{H}^{2} \cdot\right.$ Songtao $\mathrm{Guo}^{2} \cdot$ Baoguo $\mathrm{Li}^{2,3}$ \\ Published online: 30 April 2021 \\ (c) Deutsche Gesellschaft für Säugetierkunde 2021
}

Correction to: Mammalian Biology

https://doi.org/10.1007/s42991-021-00116-3

In the article, Youlatos et al. (2021). Positional behavior, habitat use, and forelimb morphology of Père David's Rock Squirrels Sciurotamias davidianus (Milne-Edwards, 1867) (Sciuridae, Rodentia) in the Qinling Mountains, Shaanxi, China (https://doi.org/10.1007/s42991-021-00116-3), collection information on the specimens used in our analyses was missing. This correction updates the article by presenting the missing information in the Appendix.

The original article has been corrected.

\section{Appendix}

Accession numbers of the studied material housed in the USMNH.

Ammospermophilus harrisii (3)—248454, 270704, 526321.

The original article can be found online at https://doi.org/10.1007/ s42991-021-00116-3.

Dionisios Youlatos

dyoul@bio.auth.gr

$\triangle$ Baoguo Li

baoguoli@nwu.edu.cn

1 Department of Zoology, School of Biology, Aristotle University of Thessaloniki, 54124 Thessaloniki, Greece

2 Shaanxi Key Laboratory for Animal Conservation and College of Life Sciences, Northwest University, Xi'an 710069, Shaanxi, China

3 Institute of Zoology, Shaanxi Academy of Sciences, Xi' an 710032, Shaanxi, China
Ammospermophilus leucurus (7)—41396, 54161, 93712, 398261, 510859, 531440, 564090.

Callosciurus erythraeus (3)—253311, 254584, 255936.

Callosciurus erythraeus bonhotei (4)-253309, 253310, 254808, 255368.

Callosciurus finlaysonii (1)—267366.

Callosciurus notatus (1)—548412.

Callosciurus prevostii (3)—259034, 396642, 545016.

Cynomys gunnisoni (5)—133695, 448236, 448238, 485422, 533015.

Cynomys ludovicianus (10)—21659, 21660, 23541, 35084, 35086, 34976, 261275, 266930, 491380, 564088.

Funambulus pennantii (3)—328002, 328003, 395769.

Funisciurus isabella (3)—539406, 539407, 539408.

Funisciurus lemniscatus (2)—539410, 539411.

Funisciurus pyrropus (2)—539421, 539422.

Heliosciurus rufobrachium (3)—543109, 539427, 539431.

Marmota caligata (3)—271698, 271701, 583155.

Marmota caudata (2)—173381, 173386.

Marmota himalayana (3)—198637, 198638, 255958. 
Marmota monax (5)—297840, 349711, 397378, 514747, 514748.

Protoxerus stangeri (4)—237333, 539438, 539443, 543111.

Ratufa affinis (2)—151757, 317202.

Ratufa bicolor (2)—49703, 261070.

Ratufa indica (2)—155401, 308415.

Ratufa macroura (1)-269970.

Sciurotamias davidianus (9)—258507, 258508, 258509, 258511, 258512, 258513, 258514, 258516, 259735.

Sciurus aberti (7)—448232, 448233, 448234, 448235, 548403, 548404, 548405.

Sciurus aestuans (2)—548445, 548446.

Sciurus carolinensis (12)—248250, 347937, 347938, 347939, 347948, 396996, 397042, 397099, 397180, 397182, 397211, 541441.

Sciurus niger (12)—264948, 397092, 397093, 397094, 397118, 397120, 397121, 397166, 548037, 548043, 548045, 548047.

Sciurus variegatoides (8)—449011, 449012, 449014, 449015, 449016, 449017, 449018, 449019.

Sciurus vulgaris (3)—581893, 582904, 582905.

Spermophilus columbianus (7)—398222, 398328, 398292, 398301, 398304, 398562, 564095.
Spermophilus lateralis (10)—271150, 398243, 398305, 398306, 398307, 398309, 398310, 398311, 501002, 564113.

Spermophilus major (3)—273183, 273184, 273185.

Spermophilus richardsonii (10)—192877, 552453, 552454, 552455, 552456, 552457, 552458, 552460, 552461, 552467.

Spermophilus spilosoma (3)—98631, 192770, 497850.

Spermophilus tridecemlineatus (9)-HW5001, HW5002, 47612, 348373, 398257, 399295, 541440, 564105, 564106.

Spermophilus undulatus (8)-135159, 246459, 247005, 251530, 251531, 251532, 251533, 397001.

Spermophilus variegatus (3)—55248, 498437, 501001.

Tamias striatus (12)—49823, 261015, 347165, 347967, 348380, 349625, 397128, 397132, 397136, 398269, 505613, 569012 .

Tamiasciurus hudsonicus (12)—397012, 397071, 397090, 397097, 397145, 397148, 397150, 397153, 397154, 397155, 397163, 397175.

Publisher's Note Springer Nature remains neutral with regard to jurisdictional claims in published maps and institutional affiliations. 